\title{
The Teaching of the Concept of Derivative in High School and Its Relationship with Physics
}

\author{
Le Thi Hoai Chau ${ }^{1}$, Ngo Minh Duc ${ }^{1}$, Duong Huu Tong, ${ }^{2, *}$ \\ ${ }^{1}$ Department of Mathematics, Ho Chi Minh City University of Education, Ho Chi Minh City, Vietnam \\ ${ }^{2}$ School of Education, Can Tho University, Can Tho City, Vietnam
}

Received November 26, 2020; Revised December 25, 2020; Accepted January 28, 2021

\begin{abstract}
Cite This Paper in the following Citation Styles
(a): [1] Le Thi Hoai Chau, Ngo Minh Duc, Duong Huu Tong, "The Teaching of the Concept of Derivative in High School and Its Relationship with Physics," Universal Journal of Educational Research, Vol. 9, No. 1, pp. 186 - 201, 2021. DOI: 10.13189/ujer.2021.090121.
\end{abstract}

(b): Le Thi Hoai Chau, Ngo Minh Duc, Duong Huu Tong (2021). The Teaching of the Concept of Derivative in High School and Its Relationship with Physics. Universal Journal of Educational Research, 9(1), 186 - 201. DOI: 10.13189/ujer.2021.090121.

Copyright $\odot 2021$ by authors, all rights reserved. Authors agree that this article remains permanently open access under the terms of the Creative Commons Attribution License 4.0 International License

\begin{abstract}
The purpose of this study was to give students a complete understanding of the derivative concept and at the same time help them to realize the critical applications of derivatives in many problems of physics, from which students see the close connection of mathematics and physics. Because this was a case study, the sample included only 30 grade 11 students in $\mathrm{Ho}$ Chi Minh City in Vietnam. The instrument included physics problems designed to show students the derivative development associated with some situations in physics. Reliable data were obtained, including students' worksheets, teacher conversations, and they were analyzed qualitatively to clarify students' understanding of derivative concepts and problem-solving abilities. It was reported that once the two-increase ratio boundary defined the derivative, the students involved in experiments realized the general significance of the derivative in calculating the instantaneous quantity change rate. This interdisciplinary approach also promoted the use of derivatives in many different contexts of science and practice, particularly in mathematics and physics.
\end{abstract}

Keywords Derivative, Students' Worksheets, Teacher Conversations, Change Rate, Mathematics, and Physics

\section{Introduction}

Interdisciplinary teaching is to identify knowledge contents that have similarities to two or more subjects to teach to avoid students having to repeat the same knowledge content in many different subjects. For interdisciplinary knowledge with the dominant subject, it is possible to arrange teaching in that subject's program and not be re-taught in the other subjects. There are several advantages of integrated teaching for students. The content with more objective practicality helps the lesson become lively, attractive to them, not dull, but motivates them to create and think according to their thinking styles. The knowledge that they apply immediately can solve real-world problems with little rote learning. Besides, the integrated content also saves students time to learn other knowledge because they do not have to repeat the same content in different subjects. That not only creates too much pressure, causes tedious learning, slows down their ability to think, turns the brain into programmable machines, but instead increases self-awareness, mastery learning activities, helping children regain interest. Also, interdisciplinary integration offers some benefits for teachers. Teachers understand interdisciplinary knowledge in their subject teaching, so it is easy to synthesize and reduce knowledge into main ideas that are easy to visualize and do not overlap. Moreover, teachers are not merely transmitters of knowledge, they also self-organize, examine, evaluate, and orient learning for students inside and outside the classroom with this method. Additionally, teachers of relevant subjects will have more favorable conditions and be more proactive in coordinating and 
supporting each other in teaching.

Calculus has great practical power. This subject manifests itself in its diverse and influential applications in practice and in many fields of science, especially physics, which have been most closely related to analysis throughout history. Even according to Kleiner [24], calculus is the primary quantitative tool for studying scientific problems in the last three centuries (...), without it, then modern physics and engineering would not exist. Therefore, calculus cannot focus only on solving pure mathematical problems and ignore the opportunity to help learners see the important instrumental role of calculus. From the cognitive perspective, the construction of an idea or notion is made from successive searches of similarities and differences with what the student already knows to make a generalization then [25].

Many studies show that learners have difficulty applying the calculus knowledge they learned in the math class to solve physics tasks. For example, according to the investigation by Redish et al. [30], although many students could use their analytical knowledge to solve mathematical problems successfully, they did not do the same thing in the context of physics. Jones's study $[19,20]$ confirmed that it seemed that learners' math knowledge had not been successfully activated in science classes. The problem was not a lack of knowledge. For example, as Bajracharya and Thompson [1], or N. M. Duc [12], even with sufficient knowledge of mathematics and physics necessary, learners still have difficulty connecting; they understand physics problems with a calculus tool. Many students did not know what the application of analytical knowledge they learned in physics, when, and why it was used in those problems (López-Gay, Sáez \& Torregrosa [25]). Explaining this phenomenon, Jones [19] stated that the reason laid in the calculus courses successfully provides students with a form of knowledge and the form needed to solve the math class tasks. However, not preparing for the successful use of this knowledge in science classes.

Two main issues need to be retained from the analysis above. Firstly, it is necessary to find a teaching method that can help students fully understand the conceptual nature and become familiar with analysis techniques. Second, it is necessary to make students apply the knowledge of analysis to physics or understand the various calculus applications appearing in the high school physics program. What solution helps to achieve these two goals? Many educational researchers examined the formation and evolution of calculus in history to find more suitable teaching approaches (Kaput [21]; L. T. H. Chau [7]; Doorman \& Van Maanen [9]; Bressoud [6]). Historical analysis shows a strong relationship between calculus and the dynamics of practice and the sciences. In particular, there has been a very close bond between analysis and physics throughout history. Many of the problems posed by Physics have been the driving force behind the emergence and evolution of analysis concepts. In the opposite direction, the tools that calculus offers help physics solve many of its problems. The mutual support between these two sciences has created significant developments in the history of human civilization. Researchers thought that it should be utilized in teaching analytical knowledge in schools today.

The researchers found a solution to overcome the two above difficulties in understanding and applying calculus concepts to take advantage of the connection between mathematics and physics in the teaching process for two subjects. This research direction leads to a teaching trend, commonly known as the interdisciplinary integration of math and science. According to Berlin and White [3], this research direction was mentioned in the early 20th century and received more attention in recent decades. In the trend above, many models discussing the connection between mathematics and science subjects had been developed by researchers. In which, people often emphasized the following two main interdisciplinary interactions:

i). Mathematics - Science context (Math - Science context): science provides contexts, principles, content that give meaning and the reason for the concept of mathematics.

ii). Science - Apply Math (Science - Apply Math): emphasizing mathematics as a tool to help solve science problems.

Many studies have shown that learners have difficulty using derivatives in physics problems (Beichner [2]; Bingolbali et al. [5]; Jones [20]). According to Jones [20], the main reason is that the analytical teaching program emphasizes the geometric meaning of the derivative (slope). The application context of physics mainly used how to understand the rate of change. This mismatched coupling created a gap between learning the derivative and understanding its useful applications in physics. This statement was also supported by Dray et al. [11] when they showed that to bridge the gap between math and physics, the central idea of the derivative concept should be the rate of change rather than the slope of the tangent.

Many works on derivative teaching were associated with physics applications, but most focused on the dynamic context involving three distance, speed, and acceleration quantities. However, according to the conclusion of Thompson [1] and Bezuidenhout [4], it seemed that the dynamic context did not help students fully understand the meaning of the rate of change. Students understood that the derivative was applied to calculate velocity or acceleration without expanding the understanding of the rate of change for quantities outside the dynamic context or even the quantities that do not change time. Jones [20] concluded that overfocusing on the derivative applications in the kinetic context may not fully understand the derivative to be used in many other contexts of the derivative in physics.

N. M. Duc's study's findings [12] revealed that the 
students' knowledge about the derivatives was inadequate to understand their various applications in high school physics. Since students understood the use of derivatives in these physical problems, the derivative needed to understand the tangent of the applied curve's instantaneous change rate to approach the function derivative. The basic calculus concepts, such as derivative, were closely related to meaning with many physical quantities such as velocity and acceleration. Therefore, prior experiences and knowledge about these quantities in real life or the study of physics could help learners understand the abstract concepts of calculus better. Marrongelle's studies [26] called for the use of knowledge and context from physics to construct meaningful notions for analytic concepts such as derivatives. Firouzian and Speer [14] provided a classification scheme of physics to learn calculus concepts, including contextualization to solve problems, illustrations, language - mix, and ultimately not in use. Some other authors, while studying how teaching associates calculus with physics, proposed some theories such as "cognitive blend" (Fauconnier \& Turner [13]) or "cognitive resources" (Hammer [16]). These theoretical prisms allowed learners to understand how they use mathematics to solve physics problems or use physics insights to develop an awareness of a mathematical concept.

The review results showed a double difficulty of learners understanding derivative concepts and using them to solve physical context problems. In many cases, it was the lack of conceptual understanding that causes the difficulties in their application. Many studies showed that learners had difficulty understanding the derivative of the change rate. However, these insights were most useful for applying derivatives in various physics and practice problems. Therefore, many authors suggested a greater emphasis on the above interpretations to create a link between analytical teaching and its physics application. Other studies looked at ways and schemes that allowed physics to be used to aid in understanding analytic concepts. Some authors also called for examining the historical development of calculus to see the interdisciplinary connections between it and physics and teach in schools. The available research also showed that the research in the world about calculus teaching in general, calculus teaching in the direction of an interdisciplinary approach mainly focused on students of universities and colleges. Researches on the derivative teaching for general students in the direction of math physics inter-tissue had not received appropriate attention. Moreover, few studies in the intermodal direction considered the program content's alignment and the reciprocal effects between teaching mathematics and physics in high schools.

\section{Theoretical Framework}

\subsection{Understanding and Approach of Interdisciplinary Concepts: The Case of Interdisciplinary between Mathematics and Science}

There are three concepts of interdisciplinary approaches when considering the connection between subjects in teaching. The first way considers interdisciplinarity as a form of integration in which a close interaction between disciplines occurs (Roegiers [31]; Drake [10]; D. H. Tra [32]). The second way considers interdisciplinarity as a basic concept and uses it to study the cooperation between subjects in both epistemological and teaching aspects (D'Hainaut [8]; Jacobs [18]). The third trend recognizes the similarity between the connotations of the terms "integrated" and "interdisciplinary." However, the difference is that the interdisciplinary teaching process preserves each subject's boundaries in the process combinations (Mathison and Freeman [27]; Frykholm and Glasson [15]). Nevertheless, the researchers realize that regardless of the trend, its core characteristic is still utilizing the cohesion and coordination among the subjects in teaching to discover new knowledge or solve some common problem. Based on this core feature and synthesizing the points from the three presented trends, we give our understanding and clarify the concept of interdisciplinarity when approaching it in teaching as follows:

(1) The division's interdisciplinary concept is to cooperate between the subjects from both the aspects of discovery and the organization of knowledge and teaching methods.

(2) The cooperation above aims to bring benefits to each subject in knowledge generation and progression or solve common problems or topics.

(3) The way to achieve this goal is to combine the use of knowledge, methods, processes, or skills of many subjects, but these elements remain intact within each subject's boundaries to which they come.

With this understanding, interdisciplinary means preserving each subject's integrity in mutual support. It will be appropriate for us to approach teaching derivative concepts by taking advantage of mathematics and physics connections. Next, the researchers will discuss the interdisciplinarity between mathematics and science subjects in teaching. The connection between mathematics and the sciences such as physics, chemistry, biology has taken place throughout their long history of development. The sciences bring resources (problems, contexts, principles, objects of study) as the driving force for mathematics development. On the other hand, mathematics provides the sciences' language and tools to do their quantitative research. This connection is perfectly suitable to apply to teaching, and it makes a special for the 
interdisciplinary relationship between mathematics and science subjects in the school.

This article uses two interdisciplinary interactions (Mathematics - Science context and Science - Apply Math) to create appropriate support for each subject. Specifically, in addition to the subject's internal objectives, math teaching also needs to provide concepts, ideas, and methods to solve science problems in general and physics. In the opposite direction, some physics problems can be used to give motivation and meaning to mathematical concepts. Moreover, physics contexts and knowledge can help learners understand better math knowledge, which is abstract and often requires rigorous proof.

\subsection{Three Strategies for Interdisciplinary Mathematics and Science Teaching}

Nikitina and Mansilla [28] pointed out three interdisciplinary strategies to overcome the isolation between science and math subjects in traditional high schools. In turn, three strategies are setting up essentializing concepts, contextualizing, and building centered problems. Establishing core concepts is a strategy to elevate concepts, principles, theories in mathematics and sciences to core concepts, the foundation from which internal connections can be created uniformity in mathematics and sciences. The more core (general) a mathematical concept is, the broader the scope of application in the sciences, and many suitable connection points can be found.

Contextualization: Setting the scene for a concept or an idea is to bring it into an environment, a broader context, where it has proper and full meaning. This strategy puts mathematical and scientific knowledge in the historical context of the formation and development of ideas. Understanding the evolutionary history of a mathematical concept can help find the appropriate contexts that allow mathematics to have many closely related fields such as physics, chemistry.

Center - Problem: is a strategy for building central problems, in the sense that it is necessary to mobilize knowledge and skills of both mathematics and other sciences to solve. Knowledge of many subjects will converge on this one problem, thereby creating a favorable situation for them to be mixed. Center-problems can be sought from real life, from applying mathematical tools to the sciences, or from analyzing historical origins to see what problems motivate the formation of the concept.

To apply the strategy of establishing core concepts in our research, we need to show a general understanding of derivatives that impact solving many scientific problems in physics. Moreover, analyzing mathematical objects in history also shows the close connection between calculus and physics in history. It suggests interdisciplinary contexts that teaching can utilize to generate the concept of derivatives and give it full meaning. Additionally, the study of some useful applications of derivatives in high school physics programs can provide suggestions to build the center- problems in which students must combine mathematics and physics knowledge to solve.

\subsection{The Concept of Derivative in the Context of Vietnam}

Before appearing officially in physics textbooks, the derivative was implicitly presented by the authors as a tool to study the instantaneous rate of change of a quantity over time. After this concept was introduced in 11th grade, physics textbooks $[22,23]$ and students used it explicitly and still associated with the derivative's interpretation in terms of change rate. There are two things from above. First, physics provided contexts and problems that could give rise to the derivative concept and give meaning to the instantaneous change rate. Second, physics teaching institutions need explicit derivative tools to solve the posed problems in the impact of the rate of change understanding. From an interdisciplinary perspective, we see the potential support that physics textbooks provide to understand the derivative concept better. Does the math textbook, in turn, make proper use of these interdisciplinary connections and meet the physics textbook's requirements? The answer will be clarified after the researchers analyze the relationship between the derivative in the math and physic textbooks.

Math textbook uses physics problems to create motivation for derivative concepts. The 11th-grade standard math textbook introduces the concept from two physics problems: finding instantaneous velocity and finding instantaneous current. Meanwhile, the 11th-grade advanced math textbooks [17,29] only consider determining the free-falling object's instantaneous velocity. The approach mentioned above shows that the above textbooks have taken into account interdisciplinary views when presenting the concept of derivative associated with the physical context. However, later calculus shows that this approach has not yet reaped the required interdisciplinary benefits - one of which is giving the meaning of the instantaneous rate of change to the derivative concept. It must be observed what textbooks want to achieve when introducing the two above physics problems.

Thus, the math textbook intends to explain a concept called "derivative" and is calculated by the limit of the form $f^{\prime}\left(x_{0}\right)=\lim \left[\left(f(x)-f\left(x_{0}\right)\right):\left(x-x_{0}\right)\right] ; x \rightarrow x_{0}$. The above approach will give a physical meaning to the derivative, which is the instantaneous velocity and amperage but does not highlight the derivative's general idea as a measure of the rate of change of one quantity according to another. It can not be sure that the math textbook intends to appear this general meaning, but the term "rate of change" has never been mentioned in the 
contents related to the derivative concept. The mathematical textbook gives three applications of derivatives in physics through the formulas for calculating instantaneous velocity, acceleration, and amperage such as $v\left(t_{0}\right)=s^{\prime}\left(t_{0}\right), a\left(t_{0}\right)=v^{\prime}\left(t_{0}\right)$ and $i\left(t_{0}\right)=Q^{\prime}\left(t_{0}\right)$.

The physics textbook poses problems that need derivatives to solve the effect of the instantaneous rate of change. From an interdisciplinary perspective, these are favorable contexts that math textbooks can utilize to generate and provide a complete understanding of concepts. Furthermore, understanding the change rate will help re-illuminate many situations where physics textbooks used implicit derivatives earlier. However, analyzing the presence of derivatives in math textbooks shows that understanding the derivatives as the rate of change has no chance of appearing. Although the mathematics textbook uses the physics problems as a driving force to the derivative concept, it does not raise its idea as a tool for calculating the rate of change of quantities.

Furthermore, problems related to the derivative in math textbooks emphasize computational techniques and the geometric significance of the derivative as the tangent slope. The types of tasks associated with the physical context are limited to two kinetic quantities, velocity or acceleration. The technique to deal with them is to use the provided formulas without related physical features relative to the change rate. In short, the interdisciplinary coupling between the two disciplines is only shown at the level of providing some formulas for calculating velocity and acceleration through derivatives. This level is not satisfactory when the math textbook has not made proper use of the "resources" from Physics and has not provided all the mathematical tools that physics textbooks need. This inadequacy raises the need to build a derivative teaching project that can explain the instantaneous change rate. That is also one of the essential works in this research.

\section{Objectives of the Study}

(1) Introduce students to the derivative concept to understand the instantaneous rate of change and align it with the derivative definition in terms of the ratio of two differences.
(2) Help students learn the derivative tool's role in solving physics problems and understand the general meaning of the concept behind the applications and formulas to calculate it.

\section{Materials and Methods}

\subsection{Participants}

The experimental class included 30 students of class 11.1 of Ho Chi Minh City University of Education Practical High School. These were natural class students' outstanding academic ability in mathematics. The time of the experiment occurred when the children had just learned through the limit of a function.

\subsection{Instruments}

Four problems had been selected to build a way to understand the instantaneous rate of change for the derivative concept. The last problem to re-illuminate derivatives applications in many problems of the high school physics program appeared earlier.

Problem 1. A microbial culture vessel is kept at $0^{0} C$. At the initial time, $t=0$ it is the heat supplied. In the first and last period, a function's flask temperature is calculated, which increases gradually: $f(t)=(t-1)^{3}+1$ where $f(t)$ (in degrees Celsius) is the culture vessel's temperature at $t$ seconds.

a). Calculate the average rate of increase in the culture vessel's temperature above between time $t_{0}=0.5$ seconds and then 1 second $\left(\Delta t=t-t_{0}=1\right)$.

b). Calculate the average rate of increase in the culture vessel between time $t_{0}{ }^{\prime}=1.25$ then 1 second $(\Delta t=1)$.

c). At two times $t_{0}=0.5$ and $t_{0}{ }^{\prime}=1.25$, when do you think the temperature of the culture flask increases faster?

d). Please suggest different ways to check which of these two times the culture bottle's temperature rises faster.

Problem 1 '. The left figure below is the functional graph $f(t)$, and the right is the enlarged image of the graph at $t_{0}=0.5$ and $t_{0}{ }^{\prime}=1.25$. 

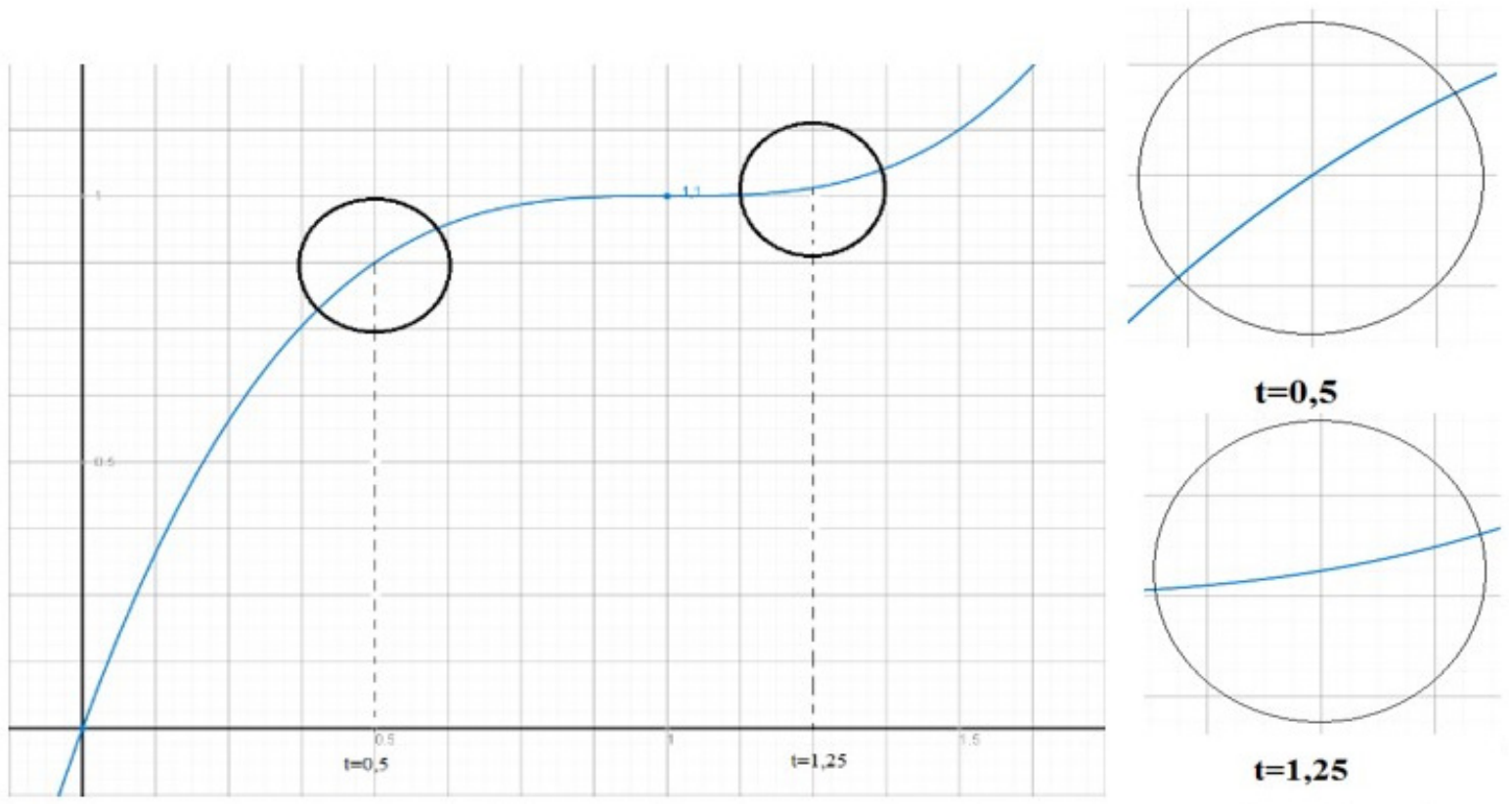

Observe and answer the question: in your opinion, at times $t_{0}=0.5$ and $t_{0}{ }^{\prime}=1.25$, when does the temperature of the culture flask increase faster? Please explain your answer.

\section{Problem 2.}

a). For two times $t_{0}=0.5$ and $t_{0}{ }^{\prime}=1.25$ find an appropriate period $\Delta t$ such that the average rate of temperature increase in the interval $\left[t_{0} ; t_{0}+\Delta t\right]$ will become greater on the segment $\left[t_{0}{ }^{\prime} ; t_{0}{ }^{\prime}+\Delta t\right]$ (instead of being smaller as we chose $\Delta t=1$ in problem 1).

b). How should these time intervals $\Delta t$ be chosen so that the average rate within these intervals more accurately describes the instantaneous rate at the moment in question?

\section{Problem 3.}

a). Calculate the average rate of temperature increase of the culture flask from the time $t_{0}=0.5$ to $t_{0}=0.5+\Delta t$ (calculation results depend on $\Delta t$ ).

b). Calculate the instantaneous temperature increase rate at the time $t_{0}=0.5$ above.

\section{Problem 4.}

a). Let the function represent velocity and acceleration in time, respectively: $v(t)$ and $a(t)$. In the 10th-grade Physics Textbook, we know that the instantaneous speed change rate is accelerating over time. Acceleration characterizes how quickly and slowly, the speed varies. Besides, to calculate the magnitude of rapid acceleration in the 10th-grade Physics Textbook, we give the following formula

\section{$|\Delta v: \Delta t|$ with tiny $\Delta t$.}

In your opinion, to calculate the exact instantaneous acceleration at a time, what is the correct formula? Explain your proposal with at least two different arguments.

b). The following statement is quoted in the article "Faraday's Law on electromagnetic induction" in 11 Physics Textbook (p.186).

“... the magnitude of the induced electromotive force in the closed circuit is proportional to the change rate of the magnetic flux through the circuit. If $\Delta t$ is small enough, the magnetic flux through the circuit changes the amount $\Delta \Phi$, then the rate of change is $|\Delta \Phi: \Delta t| "$.

Grade 11 physics textbook gives the formula $e_{c}$ for calculating the magnitude of the induced electromotive force as follows: $\left|e_{c}\right|=|\Delta \Phi: \Delta t|$, with $\Delta \mathrm{t}$ small enough. If the sign of $e_{c}$ (according to Len-xo's law) is taken into account, the formula for determining the electromotive force induced at a time is written as the following: $e_{c}=-(\Delta \Phi: \Delta t)$, with $\Delta t$ small enough.

In your opinion, has the above formula helped determine the electromotive force induced at each moment? Let us give a formula that can determine the instantaneous electromotive force induced mentioned above. Explain your proposal with at least two different arguments.

c). In the introduction, the standard 11th Grade Physics Textbook states the law of The self-induced electromotive force in a circuit: "The self-induced electromotive force is of magnitude proportional to the rate change of the current in the circuit." Accordingly, the self-induced electromotive force is 
determined by the following formula: $e_{t c}=-L \times(\Delta i: \Delta t)$ where $L$ is the inductance and $\Delta t$ is very small.

In your opinion, to calculate the exact self-induced electromotive force at a time, what is the correct formula? Explain your proposal with at least two different arguments.

\section{Possible solutions for the students}

Before asking to solve problem 1, the students are introduced to the formula for calculating the average rate of change for a function $f(x)$ when the $x$ changes its value from $x_{1}$ to $x_{2}$, which is the ratio

$$
\left[f\left(x_{2}\right)-f\left(x_{1}\right)\right]:\left(x_{2}-x_{1}\right)=\left[f\left(x_{1}+\Delta x\right)-f\left(x_{1}\right)\right]: \Delta x
$$

With this formula, students will quickly calculate the average temperature increase in sentences $a$ and $b$ of problem 1. Here, the researchers only present the strategies that may appear in solving sentences $c$ and $d$ of this problem.

i) The strategy of using the average rate of change for comparison:

Step 1: Select the time $\Delta t$ that is small enough.

Step 2: Calculate the average rate of increase over the periods $\Delta t$ for two times $t_{0}$ and $t_{0}{ }^{\prime}$.

Step 3: Compare these two average rates and conclude.

ii) The strategy of using the instantaneous rate of increases for comparison:

Step 1: Calculate the instantaneous rate of increase at $t_{0}=0.5: \lim [(f(0.5+\Delta t)-f(0.5)): \Delta t] ; \Delta t \rightarrow 0$.

Step 2: Calculate the instantaneous rate of increase at $t_{0}{ }^{\prime}=1.25: \lim [(f(1.25+\Delta t)-f(1.25)): \Delta t] ; \Delta t \rightarrow 0$.

Step 3: Compare these two instantaneous rates of increase to conclude.

iii) The strategy of using graphs:

Step 1: Observe the graph at the two times mentioned above to see when the graph is "steeper" (the tangent at the point in question has a greater slope).

Step 2: From that conclusion, the temperature increases faster.

\subsection{The Process of the Lesson Plan}

The teaching process consists of six phases, with an estimated time of 90 minutes. The class is divided into groups of 4-5 students each. In each stage, students in groups will be given a specific worksheet.

Phase 1: (15 minutes): The teacher takes a few minutes to introduce the concept of the average rate of change of a function $y=f(x)$ in the interval $\left[x_{1} ; x_{2}\right]$ is the ratio $\left[\mathrm{f}\left(\mathrm{x}_{2}\right)-\mathrm{f}\left(\mathrm{x}_{1}\right)\right]:\left(x_{2}-x_{1}\right)=(\Delta y: \Delta x)$. The groups were then delivered worksheets that contained the contents of three sentences $a, b, c$ of problem 1 and conducted discussions to solve these requirements. After the working time, each group sent a member to present their solution strategy and the results when the temperature increased faster. Other groups are encouraged to give comments and criticism.

Phase 1's goal: The idea of this phase is to create a problematic situation where the average speed over the intervals under consideration does not correctly reflect the instantaneous rate of increase at each respective moment. However, the students still do not realize their mistakes and therefore have no incentive to find another solution. The teacher will do this work in phase 2 .

Phase 2: After finishing phase 1, the teacher asks question $d$ : "propose different ways to check the time when the temperature rises faster" and invites groups to discuss and raise their hands. The teacher can suggest by asking the question, "Have you ever encountered the requirement to compare the rate of growth of a quantity in other subjects?" The teacher expects to have a group show about a method to use graphs; otherwise, it will suggest and explain this strategy's reasonableness. Right after that, the teacher gives each group a worksheet with problem 1'. In the problem, the graph of the function $f(t)$ and the image enlarged in the neighboring two points $t_{0}=0.5$ and $t_{0}{ }^{\prime}=1.25$; students are asked to compare the rate of temperature rise at these two points. The teacher and class agreed to the following conclusion: "at small intervals around two times $t_{0}=0.5$ and $t_{0}{ }^{\prime}=1.25$, the graph is an almost straight line and around the time $t_{0}=0.5$ this line is steeper, so the temperature at that time increases faster." The teacher suggests groups to compare these results with the results they obtained in problem 1 according to the change strategy's average rate.

\section{Phase 2's goal:}

The question of comparing growth rate, in our opinion, is quite familiar to students; specifically, this type of task has appeared in some lessons about population growth in geography. Moreover, in population change charts, students often rely on the line's slope connecting the points to assess which period population increases faster. In this phase, by asking to find other ways to test when the temperature rises faster, the researchers believe that the graphing approach can be suggested by the students' consensus when the teacher recommended it. However, because students participating in the experiment have not yet learned about the survey and graph of degree 3 functions, in problem 1', the researchers provide pre-drawn graphs and enlarged images at two points in time. This activity makes it easier for them to see when the temperature rises faster (steeper graph) and check the average rate of change calculation mistakes. Expected students will realize that the average growth rate may not correctly reflect the instantaneous growth rate at each time, depending on the selected time. Furthermore, that is also the engine for the next operation in phase 3 .

Phase 3: (15 minutes): The teacher hands out a questionnaire with the question of problem 2, which ask 
groups to find back an appropriate amount of time $\Delta t$ so that the average growth rate above this time is at two points $t_{0}$ and $t_{0}{ }^{\prime}$ which are consistent with the conclusion found in problem 1'. Each group performs the necessary calculations on the study sheets and then returns them to the teacher. Moreover, the corresponding average growth rate is summarized and presented to the teacher's board. The teacher allows students time to observe and ask question $b$ of problem 2: "How long should the time $\Delta t$ be chosen so that the average growth rate on which the growth rate is described more accurately time at each moment being considered? ". The teacher invites groups to speak and discuss with the whole class to draw comments: the smaller $\Delta t$ must be chosen, the more average rate of increase will reflect the instantaneous rate of change.

Phase 3's goal: The purpose of phase 3 is for students to realize that when the time interval $\Delta t$ becomes smaller and smaller (towards 0), the average growth rate will accurately reflect the instantaneous growth rate at the time being considered. However, at this time, students have not yet reached a definite value that can characterize the instantaneous rate of increase, or in other words, what the limit the researchers are talking about has not been found. In the next phase, the researchers will find a way to organize the action to emerge.

Phase 4: (10 minutes): The teacher sets up a small contest named: "Find the average rate of increase that most accurately reflects the instantaneous rate at the time $t_{0}=0.5$. " Ask each group to propose a value for $\Delta t$ and then calculate the average rate over that time interval. The group that obtains the most accurate results of the instantaneous rate of temperature increase at the moment in question will win. Teachers allow students to calculate with an existing pocket calculator or even use the calculation software installed on mobile phones. After 5 minutes of working, the teacher records each group's results on the board and shows that the winner is the group that chose the smallest interval and still calculated the average rate above that range. The teacher lets the whole class observe the groups' calculation results and realize that the average speed will reach a particular value when $\Delta \mathrm{t}$ gets smaller and smaller (approaching 0). From here, the teacher poses the discussion question: "How to get a value that accurately reflects the instantaneous temperature rise rate at the time $t_{0}=0.5$ ?". The teacher expects students to realize the need to calculate the limit $(\Delta f: \Delta t) ; \Delta t \rightarrow 0$; otherwise, the teacher suggests that students agree with this proposal and then ask the whole class to predict the above limit.

Phase 4's goal: By organizing the game looking for an average rate that most accurately reflects the instantaneous rate, the researchers encouraged groups to choose minimal intervals $\Delta t$ and calculate the average rate of increase above the selected interval. Accordingly, each group's results will be very close together and gradually become a determined value that the whole class can easily predict. This activity helps students realize that there may be an absolute limit value in their average value strategy when they choose $\Delta t$ less and less. So, by questioning how to find a value that accurately reflects the instantaneous rate of increase, the researchers assume that the strategy of using the limit $(\Delta f: \Delta t) ; \Delta t \rightarrow 0$ can emerge. This guidance is intended to appear the natural and rational motive for the derivative notion and understand the instantaneous rate of change.

Phase 5: (15 minutes): The teacher hands out worksheets with problem 3 and asks groups to find formulas to calculate the paragraph's average growth rate $[0.5 ; 0.5+\Delta t]$ (question $3 a$ ). Next, ask "calculate the instantaneous rate of increase in temperature at the time $t_{0}=0.5$ "(question $\left.3 b\right)$. The standard strategy is to calculate the limit $[f(0.5+\Delta t)-f(0.5)): \Delta t] ; \Delta t \rightarrow 0$ and get the same results as predicted in phase 4 .

Phase 5's goal: This phase aims to specify the limit strategy to find the instantaneous temperature rise rate. For sentence $a$, the researchers ask students to formulate the formula to calculate the average growth rate on an arbitrary segment $\Delta t$, and sentence $b$ is to calculate the limit when this time approaches 0 . The consistency between calculation results in phase 5 and the prediction results in phase 4 will reinforce understanding and help students get acquainted with the derivative calculation by definition by limit.

Phase 6: (10 minutes): This is the institutional phase in which the teacher presents the conceptual definition of the derivative of the function $f(x)$ at the point $x_{0}$ (symbol $\left.f^{\prime}\left(x_{0}\right)\right)$. The teacher extended further: if a function has a derivative at every point over any range, then with a value $\mathrm{x}$ in that range, there will be a corresponding derivative value. The rule of setting each $x$ with the value $f^{\prime}(x)$ helps us to set up a function called a derivative function.

Phase 7: (15 minutes): The teacher provides study cards with printed applications in derivative physics (Problem 4). The teacher can take the time to repeat or explain some relevant physics knowledge in the problem. The teacher asks each group to complete the requirements and return the papers to the teacher. With each different physical context, the teacher emphasizes the condition "to find at least two different arguments" to explain how to determine the corresponding physical quantity. The teacher expects the groups to come up with a strategy of using derivatives under two different explanations. One calculates the quantity in terms of the differential ratio (when $\Delta t$ is minimal), and the other from the rate of change characteristic of the physical quantities is under consideration. After a discussion period, the teacher asks representatives of a particular group to present their answers and other groups to give positive or critical ideas. The teacher encourages students to propose more situations in real life that can apply derivative tools. Finally, the teacher summarizes and emphasizes the 
crucial points of the lesson.

Phase 7's goal: The purpose of this phase is to enable students to re-examine the critical applications of derivatives in many physics problems that they have encountered before. In question $4 a$, the researchers provide students with the physical meaning of acceleration, a characteristic quantity for the velocity change rate. Furthermore, the researchers repeat the 10th-grade Physics Textbook formula to calculate instantaneous acceleration $a=\Delta v: \Delta t$ with a tiny $\Delta t$. This formula of limit $(\Delta f: \Delta t) ; \Delta t \rightarrow 0$ reflects the average rate of change that students have been approached in previous phases. Besides, because phase 4 and phase 5 have appeared a limited strategy, students can find out how to accurately calculate the instantaneous acceleration through the formula and also the derivative calculation just learned.

Moreover, by asking to explain the proposed formula with at least two different arguments, the researchers expect students to give another statement to explain the impact of the derivative in the situation under consideration. This approach is because acceleration is characteristic of the rate of change of velocity. Still, a derivative is a mathematical tool for calculating the rate of change to infer that $a(t)=v^{\prime}(t)$. This problem intends to help students realize the derivative concept's impact in determining the instantaneous acceleration by two approaches: one in terms of the differential ratio and the other in the sense of change rate. This approach allows learners to connect two different understandings of derivatives in the same application context. This connection helps students better understand how the derivative is applied in Physics and helps them understand more deeply math knowledge while learning. With sentence $b$ and $c$, the researchers reintroduced the physics textbook method to calculate the induced electromotive force and the self-induced electromotive force. The first is proportional to the rate of magnetic flux rate, while the second is proportional to the current change rate. By revisiting these physics applications, students can realize the essential instrumental role of derivatives in many physics problems.

\subsection{Collecting and Analyzing Data}

The researchers divided the class into six groups; each group had five students sitting in a circle. The experimental process was supported by five teaching assistants, graduate students, and the Ho Chi Minh City University of Education students, filming the learning progress during the experiment. Specifically, each assistant was in charge of filming the discussion activities of students in a group. At the end of the class, a video camera was placed to record students' and teachers' activities during the experiment. The following three types of data were recorded for us to analyze experimental results:
1) The individual exercises of each student and the group exercises after discussing each activity phase.

2) The minutes record some notable discussions of the group members during each activity phase.

3) Minutes of teacher prompting and discussion with several groups and the whole class in each activity phase.

Reliable data were collected in chronological order of phases and analyzed qualitatively to clarify physics derivatives' problems. The researchers could evaluate somewhat of the student's ability to solve problems for the selected problems. Some of the student's worksheets had been selected to explain or illustrate the understanding or solution that students had used to deal with derivative problems that appear in physics. One reason for analyzing teacher and student conversations was to clarify students' notions of solving problems that the written product did not allow.

\section{Results and Discussion}

\subsection{Results in Phase 1}

At the beginning of this phase, the teacher took a few minutes to introduce the meaning and calculation of the average change rate of function $f(x)$ in the interval $\left[x_{1} ; x_{2}\right]$. To create a bright mind for the practical problem then, in the lecture, the teacher considered a function $f(x)$ increases (co-variable) on the segment $\left[x_{1} ; x_{2}\right]$ and asked the following suggestive questions "On the section, we have seen, the variable $\mathrm{x}$ increases by an amount $\Delta x=x_{2}-x_{1}$ and the function $f(x)$ increases by a corresponding amount $\Delta y=f\left(x_{2}\right)-f\left(x_{1}\right)$. So how to calculate the average rate of increase of the function in the paragraph under consideration?".

It was noticeable that there was a student in the class who very quickly answered: "The growth rate will divide the increase of $y$ by the increase of $x$, i.e., divide $\Delta y$ by $\Delta x . "$ This format showed that before individual students participated in the experiment, the concept of an increase or change rate seemed to exist. After the above suggestive step, teachers institutionalized by defining and calculating the average change rate of a function. With this preparation step, it was not surprising that all groups easily calculate the average growth rate required in sentences $a$ and $b$ of problem 1 . For question $l c$, as the researchers expected, six groups were all using the average rate of change strategy, and no other method appeared. Specifically, the groups used the results of the average rate of increase calculated in sentences $a$ and $b$ to conclude that "at the time $t_{0}{ }^{\prime}=1.25$ the temperature of the culture vessel increased faster." 


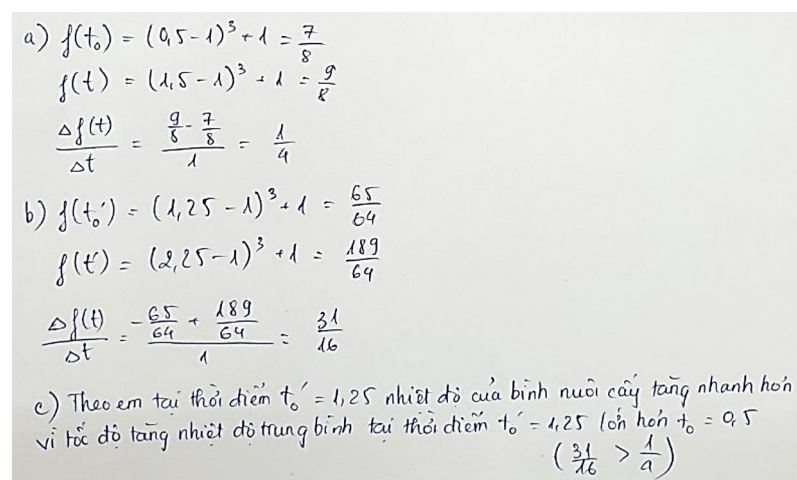

Figure 1. The solution of group 4 for problem 1

Translate the contents in Figure 1 into English:

a) $f\left(t_{0}\right)=(0.5-1)^{3}+=7 / 8$

$f(t)=(1.5-1)^{3}+=9 / 8$

$\Delta f(t): \Delta t=(9 / 8-7 / 8): 1=1 / 4$

b) $f\left(t_{0}^{\prime}\right)=(1.25-1)^{3}+1=65 / 64$

$f\left(t^{\prime}\right)=(2.25-1)^{3}+1=189 / 64$

$\Delta f(t): \Delta t=(65 / 64-189 / 64): 1=31 / 16$

c) According to me, at one point in time $t_{0}{ }^{\prime}=1.25$, the incubator's temperature increased faster because the average rate of temperature increase at one time $t_{0}{ }^{\prime}=1.25$ was greater than the time $t_{0}=0.5$ $(31 / 16>1 / 4)$.

Figure 1 showed the solution to problem 1 of group 4 . This group correctly calculated the average growth rate in sentences $a$ and $b$ and used them to make conclusions for sentence $c$. The students of these groups also stated that the reason for their decision was "the average rate of temperature increase at the time $t_{0}{ }^{\prime}=1.25$ is larger than $t_{0}=0.5$. " For the solution of group 1 , although the researchers had never given a symbol for the concept "average growth rate," students in this group used the notation $v_{t b}$ to represent it.

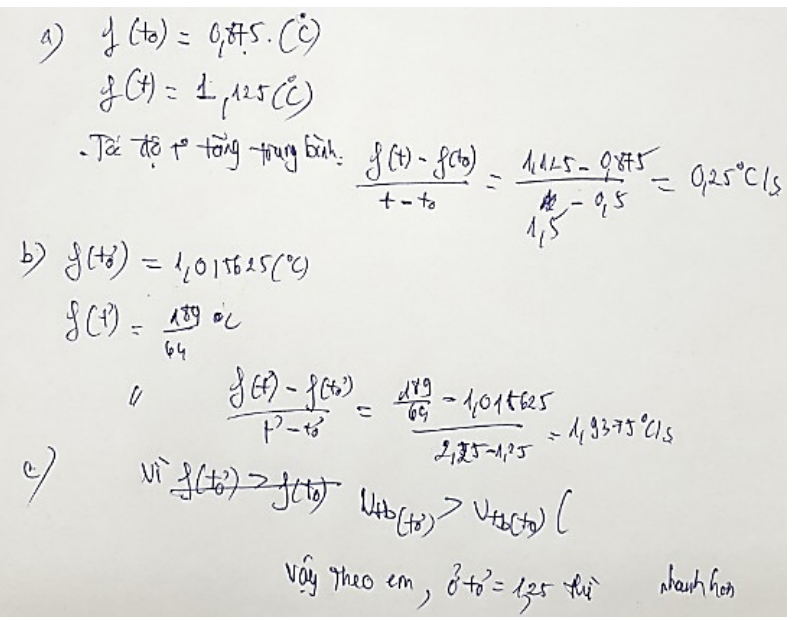

Figure 2. The solution of group 1 for problem 1

Translate the contents in Figure 2 into English: a) $f\left(t_{0}\right)=0.075\left({ }^{\circ} \mathrm{C}\right)$

$$
\begin{aligned}
& f\left(t_{0}^{\prime}\right)=1.125\left({ }^{\circ} \mathrm{C}\right) \\
& f(t)=1.125\left({ }^{\circ} \mathrm{C}\right)
\end{aligned}
$$

Average rising temperature:

$$
\begin{gathered}
{\left[f(t)-f\left(t_{0}\right)\right]:\left(t-t_{0}\right)=} \\
{[1.125-0.075]:(1.5-0.5)=0.25\left({ }^{o} C / s\right)}
\end{gathered}
$$

b) $f\left(t_{0}\right)=1.015625\left({ }^{\circ} \mathrm{C}\right)$

$f\left(t^{\prime}\right)=(189 / 64)\left({ }^{\circ} \mathrm{C}\right)$

Average rising temperature:

$$
\begin{gathered}
{\left[f(t)-f\left(t_{0}\right)\right]:\left(t-t_{0}\right)} \\
=[189: 64-1.015625]:(2.25-1.25)=1.9357\left({ }^{o} \mathrm{C} / s\right)
\end{gathered}
$$

c) $v_{t b}\left(t_{0}{ }^{\prime}\right)>v_{t b}\left(t_{0}\right)$; According to me, at one point in time $t_{0}{ }^{\prime}=1.25$, the incubator's temperature increased faster.

In Figure 2, the students argued that because $v_{t b}\left(t_{0}{ }^{\prime}\right)>v_{t b}\left(t_{0}\right)$ so at the time, $t_{0}{ }^{\prime}=1.25$ the temperature increased faster. The teacher who asked questions to students in this group about why they used the symbol $v_{t b}$ would get the answer that they related "rate of temperature increase" to the quantity "velocity" or "speed" in the physics side. This relationship of students led us to the judgment that if they understood the general meaning of a derivative, the rate of change could thoroughly apply it in the context of kinetics. Besides, considering the 10th-grade physics textbook, the researchers found that the concept of instantaneous speed was understood as the average speed in a minimal time $\Delta t$. However, suppose the group 1 students had linked "rate of temperature rise" with "speed" in mechanical motion. In that case, it seemed that the above concept of instantaneous velocity did not affect children's solution strategy. Because when asked to compare the rate of increase at each time (fast), the groups did not pay attention to whether $\Delta t$ was small enough or not but concluded when the temperature increased from an average rate of increase, results were available.

In summary, this phase's results were speculated that the concept of the rate of change or increase rate of a quantity was quite familiar to most students. Moreover, some students could even give their formula to calculate the growth rate, which was the functional increase ratio and the variable's increase when the teacher asked questions. Some students also related the rate of temperature increase with the speed or velocity in mechanical movement and used the symbol $v_{t b}$ to denote this rate of increase. However, when faced with comparing the instantaneous temperature rise rate at two points, the average rate of change strategy prevailed and led all groups to the wrong conclusion.

\subsection{Results in Phase 2}

When the teacher asked the whole class about question $c$, the students were confused. The teacher then asked 
some questions that prompted the study, and the following were recorded dialogues:

Teacher: Have you encountered any cases where the rate of increase or change rate has not been mentioned?

Students (whole class): Population growth rate, GDP growth rate, economic growth. These things are expected in geography.

Teacher: Let us give it a try; for example, talk about the population growth rate. So in geography, what do we usually do to indicate the population change over the years?

Student: Do draw the chart.

Teacher: What kind of charts do we use?

Students: There are many types of roads, columns. If we use a line chart, let us use a line chart.

Teacher: If it is a line chart, how do you know whether the population increases or decreases?

Student: If we look at the way it is pointing up, it will increase.

Teacher: So how do you know where to grow faster?

Student: Seeing steeper means increasing faster.

Teacher: In this problem, we need to know when the temperature function $f(t)$ increases faster. So what will you need?

Student: Function graph.

After this discussion, many students realized that one method to test the growth rate of that function was based on "slope." Wherever the graph was steeper increases faster, according to the students' responses, it was believed that they understood that a more vertical graph means more upward direction or a larger angle. However, since the function graph $f(t)$ was not a straight line, the correct strategy had to be based on the tangent line's inclination angle at the point under consideration. In a situation like problem 1', after the students cannot see the integral of a curve, the researchers enlarged the graph to show them the curve area. When students had observed this image, the teacher suggested that the graph was almost straight to compare their inclination angle in a relatively small area around the point being considered. The teacher asked groups to answer the question posed in problem $1 c$. The results showed that all six groups used a strategy using graphs and gave results contrary to the previous conclusions about when the temperature increased faster. For example, in the study sheets of group 4 below (Figure 3), students measured the angle created by the graph's direction with the horizontal at two times to serve as the basis for their conclusions. Specifically, in the enlarged image, they measured two inclination angles at approximately $35^{\circ}$ and $15^{\circ}$, respectively. From there, students of this group concluded that the temperature at the time $t_{0}=0.5$ increased faster because the graph was "steeper."

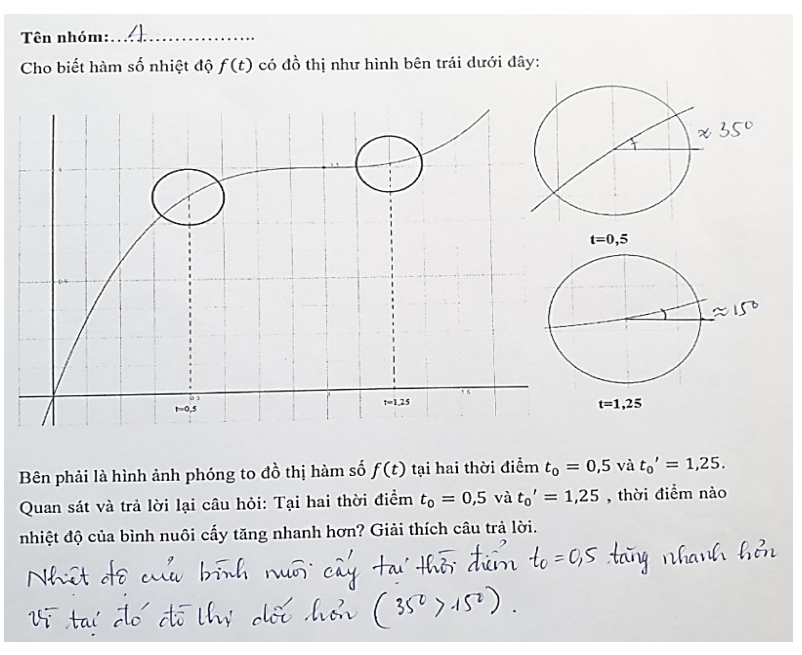

Figure 3. The solution of group 4 for problem 1'

Translate the contents in Figure 3 into English:

Observe and answer the question: in your opinion, at times $t_{0}=0.5$ and $t_{0}{ }^{\prime}=1.25$, when does the temperature of the culture flask increase faster? Please explain your answer.

The temperature of the incubator at the time $t_{0}=0.5$ increased faster because the graph was steeper there $\left(35^{0}>15^{0}\right)$.

To summarize, in this phase, it was realized that students had previous experiences with the rate of change, in particular population growth. Thus, it was easy to suggest to make the appearance of the graph strategy of finding the moment when the temperature rose faster. The graph strategy provided feedback to the groups' conclusions in problem 1 using the average rate strategy. Accordingly, all the students found the mistake that the average speed strategy made when comparing the instantaneous temperature increase rate. This situation brought a new perspective for students when they realized that the average growth rate did not necessarily reflect the rapid growth rate. A further problem posed was to find the appropriate time interval $\Delta t$ for the above reflection to be satisfied. This note was a good reason for the next activity in phase 3 .

\subsection{Results in Phase 3}

In this phase, the teacher asked the groups to reselect the time $\Delta t$ so that the average rate of temperature increased over the two segments $\left[t_{0} ; t_{0}+\Delta t\right]$ and $\left[t_{0}{ }^{\prime} ; t_{0}{ }^{\prime}+\Delta t\right]$ and reflected the correct image correlated with the instantaneous growth rate at times $t_{0}{ }^{\prime}$. The results of the groups were summarized in the table below. 
Table 1. Statistics of the results of problem $2 a$

\begin{tabular}{|c|c|c|c|c|c|c|}
\hline Groups & 1 & 2 & 3 & 4 & 5 & 6 \\
\hline $\begin{array}{c}\text { The first } \\
\text { choice }\end{array}$ & $\begin{array}{c}\Delta t \\
=1.5\end{array}$ & 3 & 2 & $<0.25$ & 0.01 & 5 \\
\hline $\begin{array}{c}\text { The second } \\
\text { choice }\end{array}$ & 0.2 & 0.1 & 0.5 & & & 11 \\
\hline $\begin{array}{c}\text { The third } \\
\text { choice }\end{array}$ & & 0.25 & & & 0.001 \\
\hline $\begin{array}{c}\text { The fourth } \\
\text { choice }\end{array}$ & & 0.1 & & & \\
\hline
\end{tabular}

According to Table 1, only group 5 was successful with the first selection out of the six groups. Most groups had to make multiple selections to find the right range. For example, in group 6, at first, they chose $\Delta t=5$ and found that they were unsuccessful, so they continued to select an enormous value $(\Delta t=11)$ and continued to fail. The group members then decided to choose a minimal time $\Delta t=0.001$ and got the right results. With group 3, although $t$ was set smaller and smaller, the third choice was still unsuccessful (when $\Delta t=0.25$ the average growth rate on the two segments was equal). Their fourth choice was not until they succeeded when choosing a small enough $\Delta t(\Delta t=0.1)$. Especially in the case of group 4, they did not try out specific values of $\Delta t$ but solved an inequality to find the condition that $\Delta t$ had to be less than 0.25 . At the end of this phase, all the groups realized that it was necessary to choose $\Delta t$ small enough to satisfy the requirement. So when the teacher summarized the whole class results on the board and asked question $2 b$, most students in the study agreed that the smaller $\Delta t$, the average growth rate in the segment đoạn $\left[t_{0} ; t_{0}+\Delta t\right]$ would reflect the growth rate more accurately.

\subsection{Results in Phase 4}

In this phase, the teacher organized the test groups to find an average rate of increase that most accurately reflected the instantaneous rate of increase at the time $t_{0}$. The teacher clearly stated that the game rules were accepted only when the groups gave a decimal value (rounding the decimal as far as possible) of the average growth rate corresponding to the chosen interval $\Delta t$. Table 2 documented six groups' results (minimal values the researchers converted to the vowel powers of 10).

All groups were aware that they had to choose a minimal $\mathrm{t}$ if they wanted to win. However, when some groups chose $\Delta t$ to be very small to calculate, a problem arose due to pocket calculators' limitations. For example, in the case of group 1, they chose $\Delta t$ to be $10^{-9}, 10^{-10}$, $10^{-11}$, the results were 0.75 . It turned out that this value was problematic, so this group only chose $\Delta t=10^{-6}$ . Moreover, obtained the average growth rate of 0.7499985 . Group 5 and group 6 still gave the result 0.75 with $\Delta t$ of $10^{-10}$ and $10^{-11}$, respectively. Group 2 had a student who had used the calculation software installed on his phone to calculate the average growth rate when $\Delta t=10^{-11}$ is 0.74999999998 . Group 4 initially intended to choose $\Delta t=0$ but then gave up because it could not be calculated. This group then proposed $\Delta t=10^{-100}$ that when the teacher asked to calculate the average growth rate, this group could not figure but still confirmed that the result would be very accurate.

After the groups reported the results, the teacher summarized them up on the board and asked the whole class about the winner. At this point, some argument arose when group 2 and group 6 chose the same value $\Delta t=10^{-11}$ but calculated different average rates. The teacher asked the whole class, then a student of group 1 stood up and answered that "the result is so close to 0.75 that normal calculators could not distinguish and round up". According to the criterion, round the decimal number as far as possible, so the teacher declared the victory belonged to group 2 with a more robust calculation tool. To conclude this phase, the teacher did the following discussion with the class:

Teacher: In your opinion, how to get a value that accurately reflects the rate of temperature increase at $t_{0}=0.5 ?$

Student: Yes, choose $\Delta t$ very small.

Teacher: But as we have seen, if the computer is strong enough, each value $\Delta t$ gives a different average growth rate? So how much is $\Delta t$ to achieve this instantaneous rate of increase?

Student: Let $\Delta t$ go to 0 , calculate the limit.

Teacher: So we predict how much this limit is?

Student: I think it must be 0.75 .

It should be added that in this activity, the researchers led the formation of a limited gender strategy according to the "approximate $x$ " approach, not choose the "approximate $f(x)$ " approach (in terms of $\varepsilon-\delta$ ). That meant creating an activity for students to realize when $\Delta t$ gradually reached the value 0 , the considered difference ratio would advance to a particular value. At the end of phase 4 , the teacher summarized and introduced the formula for calculating the function's instantaneous speed $f(x)$ at a point $x_{0}$ over the $\lim \left[\left(f\left(x_{0}+\Delta x\right)-f\left(x_{0}\right)\right): \Delta x\right] ; \Delta x \rightarrow 0$. Through this active phase, students had realized that when choosing $\Delta \mathrm{t}$ as small, the average growth rate gradually progressed to a specified value of 0.75 , and it accurately reflected the instantaneous growth rate at the time $t_{0}=0.5$. Using the limit operation that appeared logically was the best way to solve the problem posed at first. 
Table 2. The results of the contest were in phase 4

\begin{tabular}{|c|c|c|c|c|c|c|}
\hline Groups & 1 & 2 & 3 & 4 & 5 & 6 \\
\hline$\Delta t$ & $10^{-6}$ & $10^{-11}$ & 0,0006 & $10^{-100}$ & $10^{-10}$ & $10^{-11}$ \\
\hline Average growth rate & 0.7499985 & 0.74999999998 & 0.749 & $?$ & 0.75 & 0.75 \\
\hline
\end{tabular}

\subsection{Results in Phase 5}

In phase 4 , the groups predicted the target limit of 0.75 through numerical calculation. In this phase, the teacher asked the groups to calculate the instantaneous growth rate on the segment $\left[t_{0} ; t_{0}+\Delta t\right]$ according to an algebraic expression and compute the instantaneous growth rate using the limit calculation. The process of working in groups showed that algebraic computation skills were quite good. Results with up to five performance groups were allowed to take the limit $[f(0.5+\Delta t)-f(0.5)): \Delta t] ; \Delta t \rightarrow 0$ and got a result of 0.75 , consistent with the previous phase's prediction (with a group of wrong calculations). For example, Figure 4 showed an assignment of a student in group 5:

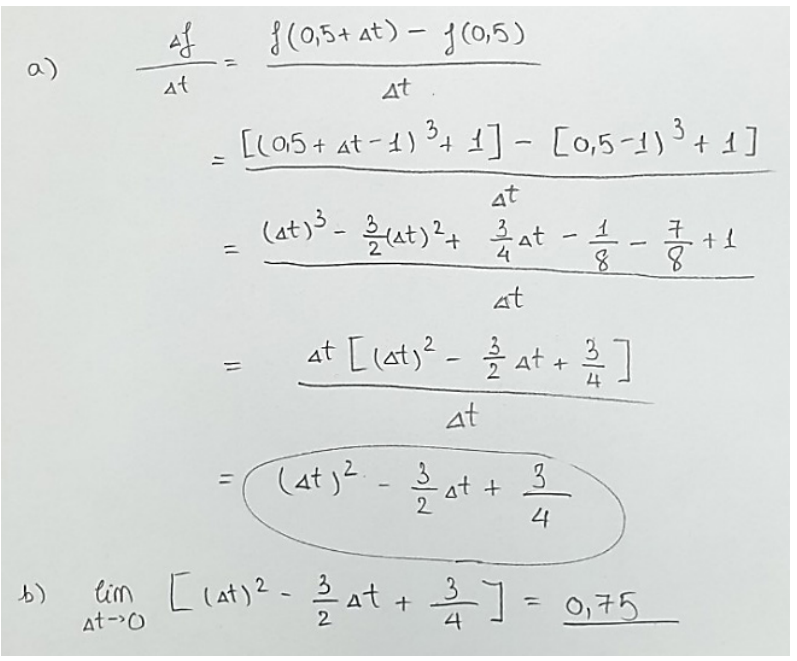

Figure 4. The solution of group 5 for problem 3

The teacher summarized the solution on the board. The obtained answer entirely coincided with the predicted limit value in phase 4 . The teacher reiterated that the limit result just found was a value that accurately reflected the instantaneous rate of temperature increase at the time $t_{0}=0.5$.

\subsection{Results in Phase 6}

This stage was the teacher's institutional phase to give an explicit definition of a concept called derivative. First, the teacher reiterated that in the above problem, when $f(t)$ describing the temperature of the culture vessel over time, the (if present) temperature helped us calculate the temperature's instantaneous change rate $t_{0}$. Since $\Delta t=t-t_{0}$ we have: $\lim \left[\left(f\left(t_{0}+\Delta t\right)-f\left(t_{0}\right)\right): \Delta t\right] ; \Delta t \rightarrow 0$ $=\lim \left[\left(f(t)-f\left(t_{0}\right)\right):\left(t-t_{0}\right)\right] ; t \rightarrow t_{0} \quad$. The teacher suggested, in general, if a particular quantity $y$ depended on variable $x$ by function $f(x)$, then $\lim \left[\left(f(x)-f\left(x_{0}\right)\right):\left(x-x_{0}\right)\right] ; x \rightarrow x_{0}$ (if any) also helped to calculate the rate of change of the function $f(x)$ by the variable $x$ at the point $x_{0}$. This limit was also written in the form $\lim \left[\left(f\left(x_{0}+\Delta x\right)-f\left(x_{0}\right)\right): \Delta x\right] ; \Delta x \rightarrow 0$ with $\Delta x=x-x_{0}$. In mathematics, if any and finite, the derivative of the function $f(x)$ at $x_{0}$ was known as the above limit because of this general significance. The teacher then gave an explicit definition of the derivative concept according to the upper limit and emphasized the meaning of its variation rate once more.

If the function $f(x)$ has a derivative at any point $x$ above the interval $(\mathrm{a} ; \mathrm{b})$, then for each $x$ value in the interval $(\mathrm{a}$; b), we can determine only one derivative value $f^{\prime}(x)$. The function describing the derivative value's dependence $f^{\prime}(x)$ on the value of $x$ in the segment $(a ; b)$ was called a derivative function and denoted $y^{\prime}(x)$ or $f^{\prime}(x)$. After this institutional phase, the students were officially introduced to the derivative's definition at a point and the derivative function. Moreover, the limit of difference ratio in the derivative's definition was attached to the method that determined the instantaneous rate of change that the students found in the previous phases. Thus, the derivative understanding of the rate of change was naturally and reasonably formed as a problem-solving tool. The meaning above could usually be increased to the change rate of any amount following the variable's evolution.

\subsection{Results in Phase 7}

The purpose of phase 7 was to create conditions for students to have the opportunity to look back at previous situations in physics textbooks where the derivative had been used implicitly. In this phase, the researchers had slightly changed how the organization works (because of time conditions) compared to expected. Specifically, instead of handing the study papers to the discussion groups to respond, the teacher introduced problem 4 on the board and let the whole class discussion and gave their answers. Below was the discussion excerpt:

Teacher: According to the entire class, how does the calculation $a=\Delta v: \Delta t$, with tiny $\Delta t$, accurately determine the instantaneous acceleration?

Students (many students said): It is approximate, teacher.

Teacher: So, what is the correct formula?

Student: Must calculate the limit when $\Delta t \rightarrow 0$ to get the exact results.

Teacher: So, when there is a function of velocity, how to calculate the acceleration? 
Student 1: Derivative.

Teacher: You realize the derivative is because you have to calculate the limit, right? So is there any other method to explain why acceleration is equal to a derivative of the speed function?

Student 2: Because acceleration is the change rate of velocity, it is calculated by derivative only.

The students quickly recognized the derivative tool's impact with the remaining two physical contexts (calculate the induced electromotive force and the self-induced electromotive force) and came up with the correct formula by derivative and explained it with two arguments: one by the limit $(\Delta v: \Delta t) ; \Delta t \rightarrow 0$ and one by the rate of change of velocity function. At the end of this phase, all students participating in the experiment recognized the instrumental role in the previous physics problems they had learned related to the characteristic of change rate. They also showed that the talk's physical quantities were also calculated according to the different ratios' limit. This activity helped learners understand the various derivatives applications and could even create strong connections between different notions about this concept.

\section{Conclusions and Suggestions}

In this teaching process, the researchers used interdisciplinary contexts to design a series of learning situations that gave learners a complete understanding of the derivative concept. The experimental helped the differential calculation strategy to appear naturally and logically. Furthermore, in this context, this limit strategy allowed finding a value that accurately reflected the rate of change of temperature at each moment. Therefore, after the derivative was defined according to the limit of two increments, students participating in experiments realized the derivative's general meaning in calculating the instantaneous rate of quantities change. The experimental results showed that the students realized the bridge between mathematics and physics [11,20,28]. Moreover, this study shows the physical role in expressing students' conception of calculus concepts [26].

This interdisciplinary approach was beneficial in terms of conceptual awareness; it also supported derivatives in science and practice contexts. Specifically, most students in the experiment used both the derivative understanding of the limit and the change rate to derive the relationship's physical quantities. This result is similar to B. P. Uyen et al. [33], but they only studied biology and archeology problems. However, the research results also revealed that some students had difficulty with symbols in both the mathematical and physical domains [30].

As a result of this teaching process, several suggestions for derivative teaching in the connection between mathematics and physics are made. Textbooks need to integrate problems to help students understand derivatives to measure the rate of change of quantities. As pointed out earlier, the instrumental role of derivatives in physics is most evident in understanding the rate of change, and physics needs this understanding to explain the reason for applying derivatives in application contexts. For that reason, math educators need to understand students' conception to help them realize the instrumental role and practical strength of the knowledge in question. Besides, teachers also need to enlighten students to re-apply applications that appeared in physics-based on mathematics knowledge. It is not unfailingly possible to restructure the mathematics and physics curriculum to satisfy the maximum of the interdisciplinary features from history (this is also the institutional constraints of teaching). As mentioned, the appearance of derivative concepts in physical textbooks often comes later than their applications. In this case, one solution for learners to realize the instrumental role of the knowledge in question is to re-illuminate their applications, which appeared implicitly earlier in physics.

Specifically, physics textbooks have implicitly used the derivative concept to solve many problems that require the rate of change understanding before being taught in math textbooks. Therefore, for students to realize the diverse applications of derivatives, math textbooks can revisit the physics mentioned above problems and clarify the instrumental role of the concept in question. Furthermore, a review of these applications can help learners better understand the derivative's features and meanings and understand why and how it is applied in many problems of the derivative in physics. At that time, the teacher can create conditions for students to look back on the implicit applications of the derivative appearing in the 10th and 11th-grade physics textbooks by giving some related problems.

At the same time, teachers need to strengthen interdisciplinary problems using knowledge of both calculus and physics. These suggestions will help students see the influential instrumental role of derivatives in physics and understand the knowledge mentioned. For example, when placing a problem with an interdisciplinary context within math teaching, the teacher can add some information to the problem to provide necessary physics knowledge. The benefits of multidisciplinary education are clear, but one obstacle to consider is that calculus and physics are often not taught in parallel or close together in the two institutions of mathematics and physics. This approach makes it difficult for learners to mobilize the necessary knowledge from the two subjects to solve problems. Suppose it is not possible to design interdisciplinary topics to teach mathematics and physics together. In that case, one solution the researchers offer is to provide supplementary knowledge for each type of task depending on the subject area learning. 


\section{REFERENCES}

[1] R. Bajracharya \& Thompson, J. R. Student Understanding of the Fundamental Theorem of Calculus at the Mathematics-physics Interface. Proceedings of the 17th Special Interest Group of the Mathematical Association of America on Research in Undergraduate Mathematics Education. Denver (CO), 2014.

[2] R. J. Beichner. Testing Student Interpretation of Kinematics Graphs, American Journal of Physics, Vol.62, No.8, 750-762, 1994.

[3] D. F. Berlin \& A. L. White. The Berlin - White Integrated Science and Mathematics Model, School Science and Mathematics, Vol.94, No.1, 2-4, 1994.

[4] J. Bezuidenhout. First - year University Students' Understanding of Rate of Change, International Journal of Mathematical Education in Science and Technology, Vol.29, No.3, 389-399, 1998.

[5] E. Bingolbali, J. Monaghan \& T. Roper. Engineering Students' Conceptions of the Derivative and Some Implications for Their Mathematical Education, International Journal of Mathematical Education in Science and Technology, Vol.38, No.6, 7-19, 763-777, 2007.

[6] D. M. Bressoud. Historical Reflections on Teaching the Fundamental Theorem of Integral Calculus, The American Mathematical Monthly, Vol.118, No.2, 99-115, 2011.

[7] L. T. H. Chau \& N. M. Duc. Training Mathematics Teachers in Accordance with Teaching to Integrated Math and Science through Teaching Integration Concept, Vietnam Journal of Education, No.6, 48-53, 2019.

[8] L. D'Hainaut. Interdisciplinarity in General Education, In International Symposium on Interdisciplinarity in General Education, UNESCO, 1985.

[9] M. Doorman \& J. Van Maanen. A Historical Perspective on Teaching and Learning Calculus, Australian Senior Mathematics Journal, Vol.22, No.2, 2008.

[10] S. M. Drake. Creating Standards-Based Integrated Curriculum: Aligning Curriculum, Content, Assessment, and Instruction, Corwin Press, A SAGE Publications Company, 2455 Teller Road, Thousand Oaks, CA 91320, 2007.

[11] T. Dray, B. Edwards \& C. A. Manogue. Bridging the Gap between Mathematics and Physics. In Proceedings of the 11th International Congress on Mathematics Education, 2008.

[12] N. M. Duc. Integrated Perspective in Teaching Integral Concepts, Ho Chi Minh City University of Education Journal of Science, Vol.14, No.4, 20-28, 2017.

[13] G. T. Fauconnier \& M. Turner. The Way We Think: Conceptual Blending and the Mind's Hidden Complexities, 2002.

[14] S. Firouzian \& N. Speer. Integrated Mathematics and Science Knowledge for Teaching Framework, In Proceedings of the 18th Conference on Research in Undergraduate Mathematics Education, 2015.

[15] J. Frykholm, G. Glasson. Connecting Science and
Mathematics Instruction: Pedagogical Context Knowledge for Teachers, School Science and Mathematics, 105(3), 127-141, 2005.

[16] D. Hammer. Student Resources for Learning Introductory Physics, American Journal of Physics, Vol.68, No.S1, 52-59, 2002

[17] T. V. Hao, V. Tuan, D. N. Nam, L. V. Tien \& V. V. Yen. Algebra and Calculus 11, Education Publishing House, 2018.

[18] H. H. Jacobs. Interdisciplinary Curriculum: Design and Implementation, Association for Supervision and Curriculum Development, 1250 N. Pitt Street, Alexandria, VA 22314, 1989.

[19] S. R. Jones. Applying Mathematics to Physics and Engineering: Symbolic Forms of the Integral (Doctoral dissertation), 2010.

[20] S. R. Jones. An Exploratory Study on Student Understandings of Derivatives in Real-world, Non-kinematics Contexts, The Journal of Mathematical Behavior, No.45, 95-110, 2017.

[21] J. Kaput. Democratizing Access to Calculus: New Routes to Old Roots, Mathematical Thinking, and Problem-solving, 77-156, 1994

[22] N. T. Khoi, P. Q. Tu, L. T. Dat, L. C. Hung, N. N. Hung, P. D. Thiet, B. T. Tuân \& L. T. Tuong. Physics 10 (Advanced), Education Publishing House, 2018.

[23] N. T. Khoi, N. P. Thuan, N. N. Hung, V. T. Khiet, P. X. Que, P. D. Thiet \& N. T. Trac. Physics 10 (Advanced), Education Publishing House, 2018.

[24] I. Kleiner. History of the infinitely small and the infinitely large in calculus, Educational Studies in Mathematics, Vol.48, No.2, 137-174, 2001.

[25] R. López-Gay, J. M. Sáez, \& J. M. Torregrosa. Obstacles to Mathematization in Physics: The Case of the Differential, Science \& Education, Vol.24, No.5, 591-613, 2015.

[26] K. Marrongelle. The Role of Physics in Students' Conceptualization of Calculus Concepts: Implications of Research on Teaching Practice, In 2nd International Conference on the Teaching of Mathematics, 2010.

[27] S. Mathison, M. Freeman. The Logic of Interdisciplinary Studies, Report Series 2.33, 1998.

[28] S. Nikitina \& V. B. Mansilla. Three strategies for interdisciplinary math and science teaching: A case of the Illinois Mathematics and Science Academy, Project Zero, Havard Graduate School of Education-Interdisciplinary Studies Project, 1-21, 2003.

[29] D. Quynh, N. H. Doan, N. X. Liem, N. K. Minh \& D. H. Thang. Algebra and Calculus 11, Education Publishing House, 2018.

[30] E. F. Redish, R. N. Steinberg, \& J. M. Saul (1996). Student Difficulties with Math in Physics: Giving Meaning to Symbols, AAPT Announcer, Vol.26, No.2, 70, 1996.

[31] X. Roegiers. A Pedagogy of Integration: Skills and Integration of Learning in Teaching, De Boeck Supérieur, 2001. 
[32] D. H. Tra. Interdisciplinary Integrated Teaching Research: Requirements Set out in the Construction, Selection of Content and Teaching Organization, VNU Journal of Science: Education Research, Vol.31, No.1, 2015.
[33] B. P. Uyen, D. H. Tong \& L. T. N. Yen. A Case Study of Teaching Real-world Problems Related to Exponential and Logarithmic Equations to Develop Students' Problem-solving Competency, Universal Journal of Educational Research, Vol.8, No.11B, 6152-6165, 2020. 